\title{
Coherent structures near the boundary between excitable and oscillatory media
}

\author{
Jeremy Bellay \\ University of Minnesota \\ Department of Computer Science \\ 200 Union St. S.E. \\ Minneapolis, MN 55455, USA
}

\author{
Arnd Scheel \\ University of Minnesota \\ School of Mathematics \\ 206 Church St. S.E. \\ Minneapolis, MN 55455, USA
}

\begin{abstract}
We investigate reaction-diffusion systems near parameter values that mark the transition from an excitable to an oscillatory medium. We analyze existence and stability of traveling waves near a steep pulse that arises as the limit of excitation pulses when parameters cross into the oscillatory regime. Traveling waves near this limiting profile are obtained by analyzing a codimension-two homoclinic saddle-node/orbit-flip bifurcation. The main result shows that there are precisely two generic scenarios for such a transition, distinguished by the sign of an interaction coefficient between pulses. In both scenarios, we find stable fast fronts, unstable slow fronts, stable excitation pulses, and trigger and phase waves. Both trigger and phase waves are stable for repulsive interaction and both are unstable for attractive interaction.
\end{abstract}

Corresponding author: Arnd Scheel, scheel@umn.edu

Keywords: homoclinic saddle-node, excitable medium, pulse interaction, stability of traveling waves 


\section{Contents}

$1 \quad$ Introduction and main results $\quad 3$

1.1 Excitable and oscillatory media . . . . . . . . . . . . . . 3

1.2 The limit of the excitation pulse - setup and hypotheses . . . . . . . . 5 5

1.3 Unfolding the steep pulse - main results . . . . . . . . . . . . . 7

2 Existence - from PDE assumptions to ODE dynamics 10

2.1 The traveling-wave saddle-node . . . . . . . . . . . . . . . . . 10

2.2 The homoclinic orbit . . . . . . . . . . . . . . . . . . 12

3 Stability - following the eigenvalue $\quad 15$

3.1 Pulses and fronts - essential spectrum . . . . . . . . . . . . . 15

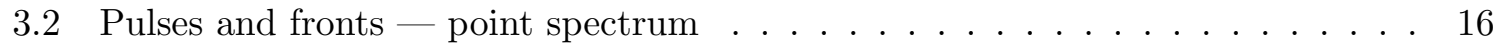

3.3 Trigger waves . . . . . . . . . . . . . . . . . . . 18

3.4 The link between excitation pulses and steep fronts . . . . . . . . . . . . . . . 19

3.5 Algebraic pulses and phase waves . . . . . . . . . . . . . . . . 21

4 Examples $\quad 22$

4.1 A scalar phase model . . . . . . . . . . . . . . . . . . . . . 22

4.2 FitzHugh-Nagumo and variations . . . . . . . . . . . . . . . 22

5 Summary and outlook $\quad 25$ 


\section{Introduction and main results}

\subsection{Excitable and oscillatory media}

Chemical and biological systems far from equilibrium are often characterized as oscillatory or excitable. Oscillatory media support asymptotically stable limit cycles. In excitable media, dynamics typically relax to equilibrium, but only after a large excursion in phase space for sufficiently large perturbations. The behavior of both types of dynamics can be represented in low-dimensional ordinary differential equations.

The simplest, scalar model describes nonlinear phase dynamics,

$$
\theta_{t}=f(\theta), \quad f(\theta)=f(\theta+2 \pi),
$$

where we think of $\theta \in S^{1}=\mathbb{R} / 2 \pi \mathbb{Z}$. If $f>0$ everywhere, the system is oscillatory. If $f$ possesses two nondegenerate zeros, the system is excitable and the distance between the two equilibria on $S^{1}$ can be taken as a possible measure for the degree of excitability. A simple specific example is $f(\theta)=\cos \theta+\mu$, oscillatory for $|\mu|>1$ and excitable for $|\mu|<1$; see Figure 1.1. A more complicated model are activator-inhibitor dynamics, such as

$$
u_{t}=f(u)-v \quad v_{t}=\varepsilon(u-v+\rho) .
$$

Here, $f$ has bistable characteristics, e.g. $f(u)=u(1-u)(u-a)$, and $\varepsilon$ is typically small. For $\rho=0$, the system is excitable: all solutions converge to $u=v=0$ as $t \rightarrow \infty$. For $\rho=-1 / 2$ and $a=1 / 2$, we find the van der Pol oscillator with a stable limit cycle - the system is oscillatory; see Figure 1.2.

In the first example (1.1), the dynamics are simple to describe for all nonlinearities $f$, and the transition from excitable to oscillatory happens as equilibria emerge on a limit cycle through a saddle-node bifurcation. At the boundary of oscillatory behavior, we find a homoclinic orbit to a saddle-node equilibrium, a typical codimension one phenomenon. In the second example (1.2), the transition from oscillatory to excitable behavior can be notably more complicated, possibly involving canards; see for instance [20].

In spatially extended systems, both oscillatory and excitable kinetics can give rise to interesting patterns. Oscillatory media support phase waves $u(k x-\omega t ; k), u(\xi ; k)=u(\xi+2 \pi ; k)$,
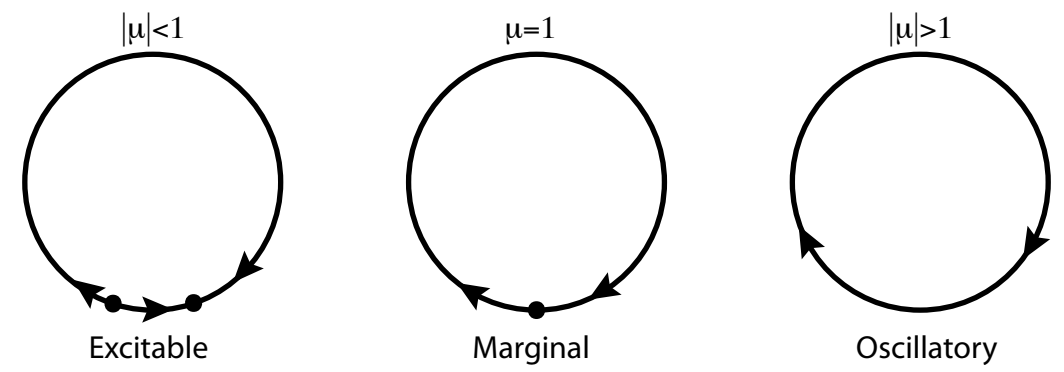

Figure 1.1: Nonlinear phase dynamics (1.1) in the excitable, marginal, and oscillatory regime. 

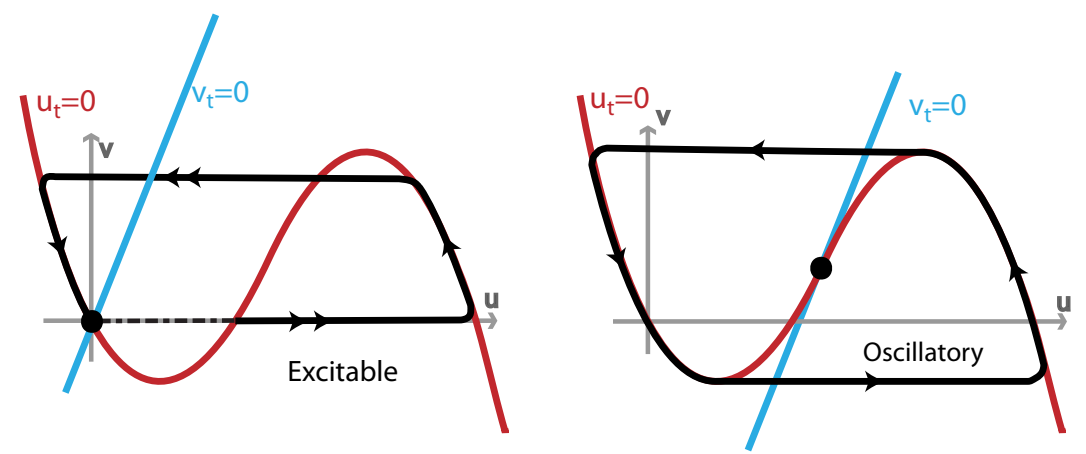

Figure 1.2: Excitable and oscillatory dynamics in activator-inhibitor kinetics.

which may nucleate at inhomogeneities or boundaries; see for instance [19]. Wave numbers vary in an admissible band, where the frequency is a function of the wave number, called the dispersion relation $\omega=\omega(k)[10,31]$. The limit $k=0$ corresponds to the spatially homogeneous oscillation. Excitable media support excitation pulses, which are emitted by wave sources such as spiral waves in two-dimensional media. Chains of excitation pulses are often referred to as trigger waves and can be similarly described by a nonlinear dispersion relation $\omega=\omega(k)$, where now $k=0$ corresponds to the single excitation pulse. A notable difference between excitable and oscillatory media is the stability of long-wavelength waves. Phase waves are always stable whenever the homogeneous oscillation is PDE stable [23, 24, 31], while trigger waves are typically either stable or unstable, depending on the sign of an interaction force between individual excitation pulses [30].

The present work is concerned with an analysis of the transition between excitable and oscillatory media. In experiments and numerical simulations, a number of striking instabilities and bifurcations have been observed for parameter values close to the transition [3, 5, 28]. Trying to understand the transition, one may start by investigating the fate of coherent structures as one approaches a critical parameter value that marks the transition. Two questions stand out from a phenomenological point of view:

- Are trigger waves and phase waves connected in parameter space?

- What happens to the excitation pulse as we cross into the oscillatory regime?

Both questions have been addressed in the scalar model (1.1) by Ermentrout and Rinzel [12]. Our goal is to answer these two questions and describe coherent structures more generally near the transition under a phenomenological assumption, rather than in the context of a specific reaction-diffusion equation. We assume that the limit of the excitation pulse exists as we converge to the boundary between excitable and oscillatory behavior, and that the limiting speed of the pulse is non-zero. We will see that this assumption is robust: the existence of a limiting excitation pulse is a codimension-one phenomenon, robust under perturbations that preserve the saddle-node bifurcation of the equilibrium. We however caution the reader that the existence of such a limiting pulse may well not be 'generic'. The excitation pulse may for instance disappear in a global bifurcation before the equilibrium disappears in a saddle- 
node bifurcation. Also, the transition to the oscillatory regime could be triggered by a Hopf bifurcation at the local equilibrium. An example for this latter scenario is the transition to oscillatory kinetics depicted in Figure 1.2, caused by shifting the nullclines of the $v$-kinetics. We refer to Remarks 2.6 and 2.7 for more precise statements.

Under a number of additional generic assumptions on this limiting pulse and the unfolding, we then describe traveling waves which are close to the pulse at each point in physical space $x$, with speed close to the speed of the limiting pulse. Our description contains existence and stability information for most of the coherent structures. It turns out that there are only two possible different bifurcation diagrams. The type of diagram is determined by the nature of the interaction between the pulses, being attractive or repulsive. In the repulsive interaction case, trigger waves have normal dispersion and are stable. In the attractive interaction case, there is anomalous dispersion and trigger waves are unstable. Our approach is model-independent, that is, our assumptions are concerned with physical properties of excitation pulses rather than specific assumptions on kinetics or diffusion coefficients.

Outline: In the remainder of the introduction, we list our main assumptions on the limiting pulse and its unfolding, Section 1.2, and state our main theorems on bifurcation diagrams, containing existence and stability information; Section 1.3. Section 2 relates our PDE assumptions to transversality in the unfolding of a codimension-two homoclinic orbit and proves the existence part of our main result by invoking a theorem of Chow and Lin [7]. Section 3 is concerned with the proof of stability and instability of coherent structures. We conclude in Section 4 with applications to specific model equations, including a nonlinear phase model (1.1), and a variant of the FitzHugh-Nagumo equation (1.2), and s brief discussion, Section 5.

Acknowledgments The authors gratefully acknowledge support by the National Science Foundation under grant NSF-DMS-0504271.

\subsection{The limit of the excitation pulse - setup and hypotheses}

We consider smooth reaction-diffusion systems

$$
u_{t}=D u_{x x}+f(u ; \mu)
$$

with $u=u(t, x) \in \mathbb{R}^{N}, D>0$ a diagonal positive diffusion matrix, and $f$ a smooth nonlinearity, depending on the parameter $\mu \in \mathbb{R}$. We are interested in traveling-wave solutions $u=u(x-c t)$, which solve the traveling-wave equation

$$
u_{\xi}=v, \quad v_{\xi}=-D^{-1}(c v+f(u ; \mu)),
$$

in phase space $(u, v) \in \mathbb{R}^{2 N}$, with independent variable $\xi=x-c t$.

Our first assumption concerns spatially homogeneous equilibria.

Hypothesis 1.1 (Saddle-node) We assume that (1.3) undergoes a generic, stable saddlenode bifurcation: 
(i) $f(0 ; 0)=0$, and $\lambda=0$ is an algebraically simple eigenvalue of $\partial_{u} f(0 ; 0)$ with eigenvector $e_{0}$ and adjoint eigenvector $e_{0}^{*}$, normalized so that $e_{0}^{*} \cdot e_{0}=1$.

(ii) $e_{0}^{*} \cdot \partial_{\mu} f(0 ; 0) \neq 0, e_{0}^{*} \cdot \partial_{u u} f(0 ; 0)\left[e_{0}, e_{0}\right] \neq 0$.

(iii) Respec $\left(-D k^{2}+\partial_{u} f(0 ; 0)\right)<0$ except for an algebraically simple eigenvalue $\lambda=0$ at $k=0$ with continuation $\lambda(k)=-d k^{2}+\mathrm{O}\left(k^{4}\right)$ for $d:=e_{0}^{*} \cdot D e_{0}>0$.

The last, somewhat less typical, assumption in Hypothesis 1.1 guarantees that the saddle-node is the only instability of the equilibrium. The coefficient $d$ is typically nonzero and necessarily non-negative for spectral stability of the critical equilibrium $u=0, \mu=0$. Hypothesis 1.1 guarantees that the kinetics $u^{\prime}=f(u ; \mu)$ possess a one-dimensional center-manifold with reduced flow

$$
u_{\mathrm{c}}^{\prime}=a_{\mu} \mu+a_{u u} u_{\mathrm{c}}^{2}+\ldots,
$$

where $a_{\mu}=e_{0}^{*} \cdot \partial_{\mu} f(0 ; 0)$ and $a_{u u}=e_{0}^{*} \cdot \partial_{u u} f(0 ; 0)\left[e_{0}, e_{0}\right]$, and $u=u_{\mathrm{c}} e_{0}+\mathrm{O}\left(u_{\mathrm{c}}^{2}+\mu^{2}\right)$. We normalize $\mu$ so that $a_{\mu} a_{\mathrm{uu}}>0$ and equilibria exist for $\mu<0$, the excitable regime, and disappear for $\mu>0$, the oscillatory regime.

Our next assumption is concerned with the existence of a pulse at $\mu=0$.

Hypothesis 1.2 (Steep pulse - existence) We assume that there exists a steep pulse at $\mu=0$ with wave speed $c_{*}>0$. More precisely, we assume that there exists a solution $u_{*}\left(x-c_{*} t\right)$ to (1.3) and $\delta>0$ such that

$$
\mathrm{e}^{\eta \xi}\left|u_{*}(\xi)\right| \rightarrow 0, \text { for } \xi \rightarrow \infty, \quad \text { and } \mathrm{e}^{-\eta \xi}\left|u_{*}(\xi)\right| \rightarrow \infty \text { for } \xi \rightarrow-\infty
$$

for all $0<\eta<\delta$. In other words, $u_{*}$ is exponentially localized in the front and decays subexponentially in its wake.

We will see in Section 2.2 that this hypothesis is actually satisfied for an open set of reactiondiffusion systems that satisfies Hypothesis 1.1. Moreover, we will see that the decay of the pulse in its wake is algebraic, $\left|u_{*}(x)\right| \sim 1 /|x|$.

We will also need an assumption on the linearization of the pulse. We therefore consider the linearized reaction-diffusion system

$$
u_{t}=D u_{\xi \xi}+c_{*} u_{\xi}+\partial_{u} f\left(u_{*}(\xi) ; 0\right) u=: \mathcal{L}_{*} u
$$

with linearized operator $\mathcal{L}_{*}$ defined on $L^{2}\left(\mathbb{R}, \mathbb{R}^{N}\right)$, with dense domain $H^{2}\left(\mathbb{R}, \mathbb{R}^{N}\right)$. We will also consider $\mathcal{L}_{*}$ in exponentially weighted spaces $L_{\eta}^{2}\left(\mathbb{R}, \mathbb{R}^{N}\right)$, equipped with the norm

$$
|u|_{\eta}^{2}:=\int\left|u(\xi) \mathrm{e}^{\eta \xi}\right|^{2} \mathrm{~d} \xi
$$

One readily verifies that $\mathcal{L}_{*}$ is closed and densely defined on $L_{\eta}^{2}$ with domain $H_{\eta}^{2}$ containing all functions in $L_{\eta}^{2}$ with second weak derivatives $u_{\xi \xi} \in L_{\eta}^{2}$. We shall later see that $\mathcal{L}_{*}$ is Fredholm of index zero for $\eta>0$, sufficiently small. 
Roughly speaking, for $\eta>0$, the $L_{\eta}^{2}$-norm enforces decay of the order $\mathrm{e}^{-\eta \xi}$ for $\xi \rightarrow \infty$, while it allows exponential growth for $\xi \rightarrow-\infty$. For $\eta<0$, the norm enforces exponential decay at $\xi=-\infty$. In particular, Hypothesis 1.2 guarantees that the steep pulse itself belongs to $L_{\eta}^{2}$ for $\eta>0$ sufficiently small, but not to $L_{-\eta}^{2}$.

Hypothesis 1.3 (Steep pulse - stability) We assume that there is $\delta>0$ such that for all $0<\eta<\delta$

$$
\operatorname{spec}_{L_{\eta}^{2}} \mathcal{L}_{*} \cap\{\operatorname{Re} \lambda \geq 0\}=\{0\},
$$

and $\lambda=0$ is algebraically simple. Moreover, we assume that $\mathcal{L}_{*}$ is invertible in $L_{\eta}^{2}$ for all $-\delta<\eta<0$.

The eigenfunction $u_{*, \xi}$, generated by spatial translation symmetry, is responsible for the kernel in $L_{\eta}^{2}$. Invertibility in $L_{\eta}^{2}$ for $\eta<0$ is possible since the steep pulse and its derivative do not decay exponentially for $\xi \rightarrow-\infty$. Again, Hypothesis 1.3 is satisfied for an open class of reaction-diffusion systems that satisfy Hypotheses 1.1 and 1.2, as we will see in Lemmas 2.4 and 2.5 .

\subsection{Unfolding the steep pulse - main results}

We will need some terminology to state our main results. We will call the solution $u_{*}\left(x-c_{*} t\right)$ from Hypothesis 1.2 the steep pulse. It will be clear later that the steep pulse is accompanied by a family of pulses that decay algebraically at both $\xi= \pm \infty$, which explains the terminology.

We say a coherent structure $u(x-c t)$ is $\varepsilon$-close to the steep pulse if $\left|c-c_{*}\right|<\varepsilon,|\mu|<\varepsilon$, and for all $\xi$ there is $\xi^{\prime}$ such that

$$
\left|u(\xi)-u_{*}\left(\xi^{\prime}\right)\right|+\left|u_{\xi}(\xi)-u_{*, \xi}\left(\xi^{\prime}\right)\right|<\varepsilon .
$$

For coherent structures close to the pulse, we distinguish the following types.

- Equilibria: equilibria are $u=0$ for $\mu=0$ and $u_{ \pm}(\mu)$ for $\mu<0$.

- Pulse: We say $u$ is a pulse if $|u(\xi)| \rightarrow u_{\infty}$ for $|\xi| \rightarrow \infty$ and some $u_{\infty} \in \mathbb{R}^{N}$.

- Excitation pulse: We call $u$ an excitation pulse if $\mu<0$.

- Algebraic pulse: We call $u$ an algebraic pulse if $|u(\xi)| \mathrm{e}^{\eta|\xi|} \rightarrow \infty$ for $|\xi| \rightarrow \infty$ and any $\eta>0$, small and fixed.

- Front: We say $u$ is a front if $u(\xi) \rightarrow u_{ \pm}$for $\xi \rightarrow \pm \infty$, with $u_{+} \neq u_{-}$. Of course, fronts close to the steep pulse can only exist in the excitable regime.

- Steep front: We say $u$ is a steep front if $\left|u_{\xi}(\xi) \mathrm{e}^{-\nu_{0} \xi}\right| \rightarrow 0, \xi \rightarrow \infty$, where $\nu_{0}=\mathrm{O}(\sqrt{|\mu|})$ is the largest root with negative real part of $\operatorname{det}\left(D \nu^{2}+c \nu+\partial_{u} f\left(u_{+}(\mu) ; \mu\right)\right)$. In other words, $u$ decays faster than the solution to the asymptotic system with weakest possible decay. 
- Fast front: We refer to fronts that are faster than the steep front as fast fronts.

- Slow front: We refer to fronts that are slower than the steep front as slow fronts.

- Small front: We say a front is a small fronts if it is $\varepsilon$-close to the equilibrium $u=0$ for all $\xi$.

- Wave train: Whenever $u$ is spatially periodic, $u(\xi)=u(\xi+L)$ for some $L>0, u^{\prime}(\xi) \not \equiv 0$, we call $u$ a wave train, $L$ its wavelength and $2 \pi / L$ its wavenumber.

- Phase waves: Wave trains are referred to as phase waves for $\mu>0$.

- Trigger waves: Wave trains are referred to as trigger waves for $\mu \leq 0$.

Theorem 1 (Bifurcation of coherent structures) Assume Hypotheses 1.1, 1.2, and 1.3 on saddle-node, existence, and stability of a steep pulse with speed $c_{*}>0$. There then is an $\varepsilon>0$ and $a C^{2}$-curve $\mu=\mu_{*}(c) \leq 0, \mu_{*}\left(c_{*}\right)=\mu_{*}^{\prime}\left(c_{*}\right)=0, \mu_{*}^{\prime \prime}>0$, and a sign $\iota \in\{+1,-1\}$ such that all solutions $\varepsilon$-close to the steep pulse are precisely the following ones:

- excitation pulses, for $\mu=\mu_{*}(c)$ and $\iota\left(c-c_{*}\right)>0$;

- steep fronts for $\mu=\mu_{*}(c)$ and $\iota\left(c-c_{*}\right)<0$;

- algebraic pulses, for $\mu=0$ and $\iota\left(c-c_{*}\right)<0$;

- trigger waves, for $\mu_{*}(c)<\mu \leq 0, \iota\left(c-c_{*}\right)>0$;

- phase waves, for $\mu>0$;

- fast fronts for $\mu<0, c>c_{\mathrm{sf}}$ if $\iota<0$, and $c_{\mathrm{ep}}>c>c_{\mathrm{sf}}$ if $\iota>0$;

- slow fronts for $\mu<0, c<c_{\mathrm{sf}}$ if $\iota>0$, and $c_{\mathrm{ep}}<c<c_{\mathrm{sf}}$ if $\iota<0$.

- small fronts and equilibria for $\mu<0$.

Here $c_{\mathrm{ep}}(\mu)$ and $c_{\mathrm{sf}}(\mu)$ are the unique speeds of excitation pulse and steep front, respectively, given through the inverses of $\mu_{*}(c)$. Phase and trigger waves are unique for each prescribed $c, \mu$. The bifurcation diagrams in the $\mu-c$-plane are depicted in Figure 1.3. Small fronts and equilibria exist in the left half, $\mu<0$, but are not included in the diagram.

Remark 1.4 The sign ı corresponds to an interaction force between steep pulses. One can formally compute an effective interaction equation between steep pulses by placing two pulse profiles at positions $x_{1}<0<x_{2}, x_{2}-x_{1} \gg 1$, with a mismatch at $x=0$, say. One then substitutes this ansatz into the reaction-diffusion equation and obtains a small error term on the right-hand side. Projecting this error term onto the kernel of the linearization at the pulse placed at $x_{1}$, using the kernel of the adjoint in $L_{-\eta}^{2}$, one obtains an effective equation for the correction to the motion of the pulse located at $x_{1}$. While this process has been made precise in terms of a weak interaction manifold [11, 22, 26, 36] for pulses on a stable background, it 




Figure 1.3: Bifurcation diagrams for $\iota>0$ (left) and $\iota<0$ (right).

is so far purely formal in our context. Nevertheless, going through this process, one would find that the attraction is interactive when $\iota=1$ and repulsive when $\iota=-1$. A somewhat explicit expression for $\iota$ is given in Remark 3.12.

We also have some information on the stability of coherent structures in the excitable regime. Note first that Theorem 1 gives precisely one type of coherent structure for each parameter value $\mu, c$ (we exclude the somewhat simpler small fronts and equilibria from our considerations, here). We denote this coherent structure by $u_{\mu, c}$. Consider now the linearized operator

$$
\mathcal{L}_{\mu, c} u=D u_{\xi \xi}+c u_{\xi}+\partial_{u} f\left(u_{\mu, c}(\xi) ; \mu\right) u,
$$

as a closed linear operator in $L_{\eta}^{2}$ for $\eta>0$. We say a coherent structure is spectrally stable in $L_{\eta}^{2}$ if the spectrum of $\mathcal{L}$ is contained in the closed left half plane.

Theorem 2 (Stability of coherent structures) We have the following information on stability of coherent structures. Fix $\eta_{*}>0$, sufficiently small. Then

- excitation pulses are spectrally stable in $L^{2}$;

- steep fronts are spectrally stable in $L_{\eta_{*}}^{2}$;

- fast fronts are spectrally stable in $L_{\eta_{*}}^{2}$;

- slow fronts are spectrally unstable for all $\eta$;

- small fronts are spectrally stable in $L_{\eta_{*}}^{2}$; 
- trigger waves are spectrally stable in $L^{2}$ when $\iota>0$ and spectrally unstable if $\iota<0$, for $\left|c-c_{\mathrm{ep}}\right|$ sufficiently small;

- algebraic pulses are spectrally stable in $L_{\eta_{*}}^{2}$ when $\iota<0$ and spectrally unstable in $L_{\eta}^{2}$ for all $\eta$ when $\iota>0$;

- phase waves with $\iota\left(c-c_{*}\right)<0$ and $|\mu|$ sufficiently small are spectrally stable in $L_{\eta_{*}}^{2}$ when $\iota<0$ and spectrally unstable in $L_{\eta}^{2}$ for all $\eta$ when $\iota>0$;

Both steep fronts and excitation pulses possess an algebraically simple eigenvalue at the origin. The spectrum of fast fronts is contained in the open left half plane, the spectrum of slow fronts contains an unstable eigenvalue of algebraic multiplicity one.

Remark 1.5 The theorem is somewhat easier to formulate using the notion of extended point spectrum; see [29]. The extended point spectrum in a neighborhood of the origin consists of a simple eigenvalue for pulses and fronts. The eigenvalue is positive for slow fronts, zero for steep fronts and excitation pulses, and negative for fast fronts. The exponential weight $\eta_{*}$ is somewhat arbitrary and used only to push the essential spectrum into the negative half plane. One can give sharp $\mu$ - and c-dependent bounds on the allowed values of $\eta_{*}$ in terms of certain eigenvalues of the traveling-wave ODE. For instance, $\eta_{*}$ needs to be sufficiently small in a $\mu$ and c-uniform fashion, but no smaller than the exponential decay rate $\nu_{0}$ of small fronts; see also Lemma 3.7.

Remark 1.6 Spectral stability implies nonlinear stability for the excitation pulses [17] and trigger waves [10]. It also implies nonlinear stability for fast and steep fronts in spaces with weight $\max \left\{\mathrm{e}^{\eta_{*} \xi}, 1\right\}$ [33]. Spectral instability of slow fronts implies nonlinear instability [17].

Remark 1.7 We note that the set of trigger and phase waves is connected. Also, stability properties coincide in the regions where we were able to determine them. The results do not give any indication of a qualitative change when one follows wave trains and crosses $\mu=0$. It is an interesting problem to investigate stability of wave trains for all admissible $\mu, c$.

\section{Existence - from PDE assumptions to ODE dynamics}

The goal of this section is to show that our PDE assumptions on equilibrium saddle-node, existence, and stability of the steep pulse homoclinic have a number of important consequences on the ODE dynamics in the traveling-wave problem (1.4). We therefore first investigate the local traveling-wave dynamics, Section 2.1, and then the homoclinic connection, Section 2.2.

\subsection{The traveling-wave saddle-node}

We start by investigating the linearization at the equilibrium $u=v=0$ of (1.4). Recall that throughout we assume $c_{*}>0$. 
Lemma 2.1 The linearization of the traveling-wave system (1.4) at the equilibrium $u=v=0$ in $\mu=0$ possesses eigenvalues $\nu_{j}, 1 \leq j \leq 2 N$, repeated with multiplicity, that satisfy

$$
\operatorname{Re} \nu_{1} \leq \ldots \leq \operatorname{Re} \nu_{N}<\nu_{N+1}=0<\operatorname{Re} \nu_{N+2} \leq \ldots \leq \nu_{2 N}
$$

Proof. The eigenvalues are roots of the dispersion relation $d(\lambda, \nu)=\operatorname{det}\left(D \nu^{2}+c_{*} \nu+\right.$ $\left.\partial_{u} f(0 ; 0)-\lambda\right)$, evaluated at $\lambda=0$. Since roots $d(\lambda, \mathrm{i} k)$ with $k$ real yield eigenfunctions to the linearization at the background state $u=0$, they correspond to essential spectrum of $\mathcal{L}_{*}[17]$ and we can conclude from Hypothesis 1.3 that $d(\lambda, i k) \neq 0$ for all $\lambda>0$. For $\lambda \gg 1$, one readily computes that roots are $\nu= \pm \sqrt{\lambda / d_{j}}+\mathrm{O}(1)$, where $d_{j}$ are the entries in the diagonal of $D$. There are therefore precisely $N$ roots $\nu$ with positive real part and $N$ with negative real part for all $\lambda>0$.

We next claim that $d(0, \mathrm{i} k) \neq 0$ for $k \neq 0$. Suppose the contrary were true, $d(0, \mathrm{i} k)=0$. This would yield a kernel $\left[-D^{2} k^{2}+c_{*} \mathrm{i} k+\partial_{u} f(0 ; 0)\right] u_{0}=0$, and therefore a zero of $d\left(-c_{*} \mathrm{i} k, \mathrm{i} k\right)$, in contradiction to Hypothesis 1.1, (iii).

We finally study $d$ near $\lambda=\nu=0$. Due to the kernel of $\partial_{u} f(0,0), d(0,0)=0$. Since the eigenvalue is simple, $\partial_{\lambda} d(0,0) \neq 0$. A short calculation shows that $\partial_{\nu} d(0,0)=-c \partial_{\lambda} d(0,0)$, so that there is a simple root $\nu=0$. Since $\partial_{\lambda} \nu=c>0$ this root moves into $\operatorname{Re} \nu>0$ for $\lambda>0$, so that there are precisely $N$ roots $\nu$ with $\operatorname{Re} \nu<0$ at $\lambda=0$. This proves the lemma.

Lemma 2.2 The traveling-wave ODE (1.4) undergoes a non-degenerate saddle-node bifurcation at $(u, v)=0$ and $\mu=0$ for any $c>0$. The equation on the center-manifold is given by $c u_{\mathrm{c}}^{\prime}=-a_{\mu} \mu-a_{u u} u_{\mathrm{c}}^{2}+\ldots$, which coincides to leading order with the time reversed equation for the pure kinetics (1.5).

Proof. One readily verifies that the kernel for the spatial dynamics linearization is spanned by $\left(e_{0}, 0\right)$ and the normalized adjoint eigenvector is given by $\left(e_{0}^{*}, c^{-1} D e_{0}^{*}\right)$, where $e_{0}$ and $e_{0}^{*}$ were defined in Hypothesis 1.1. One can now evaluate derivatives with respect to $\mu$ and $u$ on the center manifold and project with the adjoint to find the reduced dynamics on the center manifold. Since the nonlinearity enters as $\left(0,-D^{-1} f\right)$, the diffusion matrix disappears from the projection terms and we obtain the terms from the pure kinetics with an opposite sign.

Notation: Given the linearization described in Lemma 2.1, there exist manifolds tangent to generalized eigenspaces of eigenvalues with negative and positive real part, $W^{\text {ss }}$ and $W^{\mathrm{uu}}$, and to the kernel, $W^{\mathrm{c}}$. Similarly, $W^{\mathrm{cs}}$ and $W^{\mathrm{cu}}$, are invariant manifolds tangent to generalized eigenspaces of eigenvalues with non-positive and non-negative real parts, respectively. All those manifolds are smooth $\left(C^{k}\right.$ for any fixed finite $k$ ). The manifolds $W^{\mathrm{cs}}, W^{\mathrm{cu}}$, and $W^{\mathrm{c}}$ depend smoothly on parameters. The manifolds $W^{\text {ss }}$ and $W^{\text {uu }}$ depend smoothly on parameters but should merely be considered as strong stable and unstable leaves to a base point on the center manifold. The (finite) smoothness of those leaves is then limited by the smoothness of the base point. We refer to the center, center-stable, center-unstable manifold after choosing one specific representative. The non-uniqueness will not play a role in the subsequent discussions. 


\subsection{The homoclinic orbit}

Given the dynamics on the center-manifold described in Lemma 2.2, we now consider pulses that arise as intersections between $W^{\mathrm{cu}}$ and $W^{\mathrm{cs}}$ at $\mu=0$. Our requirement that the pulse decays exponentially for $\xi \rightarrow+\infty$ and subexponentially for $\xi \rightarrow-\infty$ states that it actually lies in the intersection $W^{\mathrm{ss}} \cap\left(W^{\mathrm{cu}} \backslash W^{\mathrm{uu}}\right)$. In particular, we have the typical algebraic expansion

$$
u_{*}(\xi)=\frac{c}{a_{u u} \xi} e_{0}+\mathrm{O}\left(\frac{1}{\xi^{2}}\right), \quad \text { for } \xi \rightarrow-\infty,
$$

where again $e_{0} \in \operatorname{Ker} \partial_{u} f(0 ; 0)$.

Remark 2.3 Our assumption on the decay of the pulse at the boundary $\mu=0$ can now be seen as a necessary assumption for it to be the limit of excitation pulses. If the pulse were not contained in a strong stable or strong unstable manifold, an unfolding in $\mu$ would not give pulses, only heteroclinic connections (fronts). If the intersection were between $W^{\text {uu }} \cap$ $\left(W^{\mathrm{cs}} \backslash W^{\mathrm{ss}}\right)$, we would find the analogous bifurcation diagram to Theorem 1. However, the pulses bifurcating from such a pulse that is steep in its wake rather than in the front would be homoclinic to an equilibrium with a spatial Morse index $N-1$, that is, to the PDE-unstable equilibrium. In particular, they would be PDE-unstable pulses and would not correspond to the excitation pulses that we set out to follow up to the oscillatory regime, but their background would be unstable with respect to the pure kinetics of the reaction-diffusion system.

One readily verifies that the intersection of tangent spaces $T W^{\mathrm{uu}} \cap T W^{\mathrm{cs}}$ at the steep pulse is minimal, one-dimensional, and that $T W^{\mathrm{uu}}+T W^{\mathrm{cs}}$ has codimension one. In fact, any point in the intersection would readily yield a kernel to $\mathcal{L}_{*}$ in $L_{\eta}^{2}$ for $\eta>0$, small enough. An intersection of dimension greater than one would thereby contradict Hypothesis 1.3.

In order to state the next lemma, we first note that varying $c$ close to $c_{*}$ does not change the dynamics on the center-manifold at leading order, so that we can smoothly continue both $W^{\text {uu }}$ and $W^{\text {cs }}$ in $c$.

Lemma 2.4 Assume that the eigenvalue $\lambda=0$ in $L_{\eta}^{2}$ is simple as stated in Hypothesis 1.3. Then the manifolds $W^{\mathrm{uu}}$ and $W^{\mathrm{cs}}$ cross transversely along the steep pulse upon varying $c$ through $c_{*}$. In other words, the intersection of $\bigcup_{c} W^{\mathrm{uu}}$ and $\bigcup_{c} W^{\mathrm{cs}}$ in $\mathbb{R}^{2 N} \times\{c \in \mathbb{R}\}$ is transverse.

Proof. Inspecting the variational equation

$$
u_{\xi}=v, \quad v_{\xi}=-D^{-1}\left(c v+\partial_{u} f\left(u_{*}(\xi) ; 0\right) u\right),
$$

in extended phase space, one sees that non-transversality would correspond to a solution of the generalized eigenvalue problem

$$
\mathcal{L}_{*} u=u_{*, \xi}
$$

in $L_{\eta}^{2}$. The calculation is identical to the one for pulses or fronts [31, $\left.\S 2.1\right]$ and we omit the details. One then concludes that non-transversality is excluded since the eigenvalue $\lambda=0$ was assumed to be algebraically simple. 
Lemma 2.5 Assume in addition that the kernel of $\mathcal{L}_{*}$ in $L_{-\eta}^{2}$ is trivial as stated in Hypothesis 1.3. Then the intersection $W^{\mathrm{cu}} \cap W^{\mathrm{cs}}$ is transverse along the homoclinic.

Proof. Assume the converse was true, which would yield a bounded solution to the linearized equation (2.2) that is linearly independent of the derivative of the steep pulse. Since there is precisely one solution that decays algebraically as $\xi \rightarrow-\infty$, we can add a multiple of the derivative of the steep front so that the additional solution actually decays exponentially as $\xi \rightarrow-\infty$. The $u$-component of this solution however belongs to the kernel of $\mathcal{L}_{*}$ considered in $L_{-\eta}^{2}$ for $\eta$ sufficiently small, in contradiction to Hypothesis 1.3.

Theorem 3 [7] Coherent structures in a neighborhood of the steep pulse are described by the bifurcation diagram in Theorem 1; see also Figure 2.1.

Proof. The bifurcation diagram as explained in Theorem 1 is a simple consequence of Chow and Lin's analysis of a codimension-two homoclinic bifurcation [7]. A similar result was proved by Deng [9], including also transverse and pitchfork bifurcations at the equilibrium. In fact, the results there guarantee a bifurcation diagram as depicted in Figure 2.1, up to the orientation of the diagram with respect to the parameter $c-c_{*}$, whose sign plays no distinguished role in [7]. The description in Theorem 1 is a simple interpretation of the orbits in the traveling-wave ODE as coherent structures. We need to check that the assumptions in [7] are satisfied. In fact, the assumptions there are

(i) generic saddle-node in $\mu$;

(ii) transverse crossing of $W^{\mathrm{cu}}$ and $W^{\mathrm{ss}}$ in $c$;

(iii) transversality of $W^{\mathrm{cu}}$ and $W^{\mathrm{cs}}$.

Now (i)-(iii) follow from Lemmata $2.2,2.4$, and 2.5, respectively.

Proof. [of Theorem 1] The proof is an immediate consequence of Theorem 3. The two cases $\iota= \pm 1$ are distinguished by the orientation of the diagram with respect to the parameter $c$.

Remark 2.6 We mentioned in the introduction that the existence of a limiting excitation pulse is a robust, codimension-one situation. The homoclinic bifurcation invoked here is of codimension two. However, the wave speed $c$ appears as an intrinsic rather than an external parameter and should not be counted towards codimension of the PDE phenomenon. As mentioned in Remark 2.3, it is not difficult to see that codimension-one saddle-node homoclinics would not give rise to excitation pulses. In this sense, the existence of the codimension-two steep pulse is a necessary and robust assumption that can guarantee the existence of excitation pulses up to the boundary of excitability. 


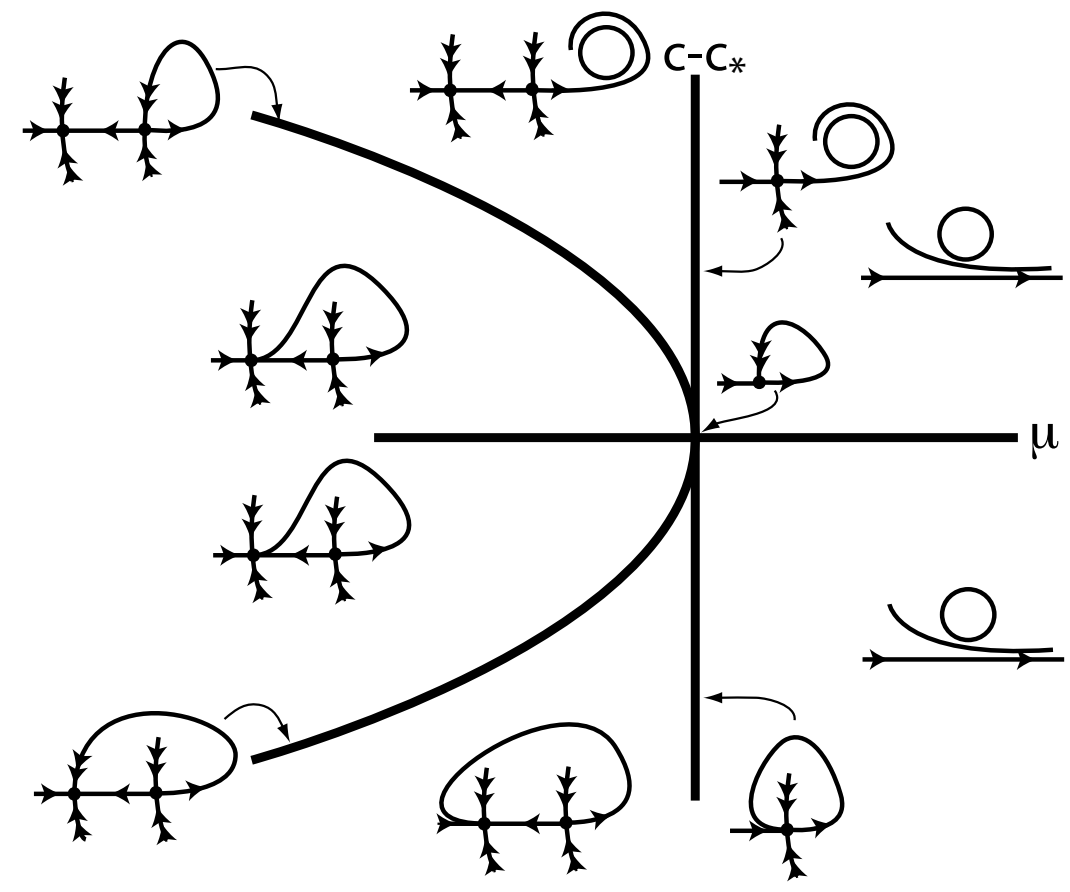

Figure 2.1: Bifurcating homoclinic, heteroclinic, and periodic orbits near the steep-pulse homoclinic as shown in [7]. The orientation with respect to $c-c_{*}$ shown is for the case $\iota>0$.

Remark 2.7 The spectral assumptions can be viewed more explicitly in at least two different ways. Splitting the linearization at the equilibrium at some real part $\operatorname{Re} \nu=-\eta$ into a generalized stable and unstable subspace, one can construct generalized stable and unstable linear bundles along the homoclinic which contain initial values to solutions with generalized decay in forward and backward spatial time $\xi$ stronger than the prescribed rate $\eta$. Our spectral assumption, Hypothesis 1.3, and Lemmas 2.4-2.5 imply that these bundles are transverse for $\eta<0$, small, and intersect along the derivative of the steep pulse for $\eta>0$, small. One can envision a codimension-three bifurcation where the homoclinic also possesses exponential decay as $\xi \rightarrow-\infty$. In this case, the bundles discussed here intersect nontrivially for $\eta<0$, too. Unfolding such a point would result in an orbit-flip, and a crossing of a zero-eigenvalue in $L_{-\eta}^{2}$. In particular, the excitation pulse would undergo a similar orbit flip and the stability of the accompanying trigger waves would change [30]. In other words, crossing of a zero-eigenvalue in $L_{-\eta}^{2}$, or, more geometrically, a flip bifurcation, marks the transition between our two bifurcation diagrams $\iota= \pm 1$. A more direct connection between the spectral and the geometric picture is provided by the Evans function [1], constructed with the help of the bundles split at $\eta<0$. In a slightly different direction, transversality of the bundles mentioned above is essential to the construction of homoclinic center-manifolds [18, 25, 35]. A flip-bifurcation induces a change in orientability of such a manifold.

Summarizing, transversality of the bundles for $\eta<0$ corresponds to generic interaction. Failure of such interaction and hence crossing of zero eigenvalues in $L_{-\eta}^{2}$ is induced by orbit or inclination flips. Similarly, changes of the sign of the interaction force $\iota$ corresponds to 
changes in the orientability of a homoclinic center-manifold.

\section{Stability - following the eigenvalue}

In this section, we prove Theorem 2 on stability. We first discuss the essential spectrum of pulses and fronts in weighted spaces, Section 3.1. We then track point spectrum of pulses and fronts, Section 3.2. In Section 3.3, we discuss stability of trigger waves. Section 3.4 connects stability information from Sections 3.2 and 3.3. Finally, Section 3.5 concludes with the spectrum of algebraic pulses and phase waves.

\subsection{Pulses and fronts - essential spectrum}

We are interested in the linearization $\mathcal{L}_{c, \mu}$ for parameter values where a front or a pulse exists. Throughout we refer to $[32,13]$ for definitions and results on the essential spectrum. We will refer to $u_{ \pm}(\mu)$ as the equilibria, so that $u_{+}$is unstable for the kinetics and $u_{-}$is stable for the kinetics. Since the flow on the traveling-wave center manifold is time-reversed when compared to the flow on the kinetics center-manifold, heteroclinic orbits all connect $u_{-}$to $u_{+}$. The dispersion relations at $u_{ \pm}$are defined as

$$
d_{ \pm}(\lambda, \nu ; \mu, c):=\operatorname{det}\left(D \nu^{2}+c \nu+\partial_{u} f\left(u_{ \pm}(\mu) ; \mu\right)-\lambda\right) .
$$

The Fredholm index of the linearization at a front in $L_{\eta}^{2}$ changes whenever $d_{ \pm}(\lambda,-\eta+\mathrm{i} k)=0$ for some $k \in \mathbb{R}[32,13]$. The zero set defines algebraic curves in the complex plane which we refer to as Fredholm borders. For $\eta=0$ and $\mu=0$, we know that $d$ vanishes along $\lambda=c_{*} \mathrm{i} k-d k^{2}+\mathrm{O}\left(k^{4}\right)$. Since equilibria $u_{ \pm}$are smooth functions of $\sqrt{-\mu}, \mu \leq 0, d$ is smooth in $\sqrt{-\mu}, \eta, c$, and we can conclude that all Fredholm borders close to the imaginary axis are given by smooth curves $\lambda=\lambda(k ; \sqrt{-\mu}, c, \eta)$, smoothly depending on parameters. Since $\operatorname{Re} \lambda=\lambda(0)-d k^{2}+\mathrm{O}\left((|\eta|+|\sqrt{|\mu|}|) k^{2}+k^{4}\right)$, we conclude that stability is determined by the sign of $\lambda(k=0)$. For $\eta=0$, we therefore find that $u_{-}$is PDE stable and $u_{+} \operatorname{PDE}$

unstable. Since $\lambda(0)=-c \eta+\mathrm{O}(\sqrt{|\mu|})$, we also see that $u_{+}$is stable for $\eta>\eta_{*}=\mathrm{O}(\sqrt{|\mu|})>0$, sufficiently small.

This shows that the essential spectrum is located in the open left half plane for choices of weights as indicated in Theorem 2.

We will from now on fix a weight $\eta=\delta$, sufficiently small, so that the essential spectrum is contained in the open left half plane for all equilibria. We will then compute the location of point spectrum in a neighborhood of the origin. The point spectrum is actually part of the extended point spectrum and as such independent of weights, as long as Fredholm borders do not cross the location of the eigenvalue; see [13, 29]. Accordingly, we will in the following refer to the point spectrum as extended point spectrum, emphasizing that it is in fact largely independent of the choice of function space. 


\subsection{Pulses and fronts - point spectrum}

We start with the following easy but crucial observation.

Lemma 3.1 The operator $\mathcal{L}_{\mu, c}$ depends continuously on $\mu, c$ as an operator from $H_{\eta}^{2}$ to $L_{\eta}^{2}$ provided that $u_{\mu, c}$ depends continuously on $\mu, c$ in $L^{\infty}$.

Proof. One readily checks that the operator norm on $L_{\eta}^{2}$ is controlled by the $L^{\infty}$-norm for multiplication operators. Therefore, both explicit dependence through the parameters $\mu, c$ in the terms $c \partial_{\xi}$ and $\partial_{u} f(u ; \mu)$ and implicit dependence through the dependence on the profile $u=u_{\mu, c}$ are continuous.

Lemma 3.2 Let $u_{\mathrm{lf}}$ be the heteroclinic within the center manifold, corresponding to a smallamplitude front between $u_{+}$and $u_{-}$. Then the linearization $\mathcal{L}_{\mathrm{lf}}$ at the profile $u_{\mathrm{lf}}$ possesses empty extended point spectrum for $\mu, c$ close to $0, c_{*}$.

Proof. For $\mu=0, c=c_{*}$, the extended point spectrum is empty in a vicinity of the origin. Lemma 3.1 then guarantees that this is true for small $\mu, c$.

Lemma 3.3 Let $\Sigma$ be the closed region in the $\mu$-c-plane which is bounded by the excitation pulse and the algebraic pulse, and which contains all front solutions. Then the point spectrum of $\mathcal{L}_{\mu, c}$ depends continuously on $\mu, c \in \Sigma$.

Proof. The statement follows from Lemma 3.1 with the exception of continuity at the excitation pulses. The excitation pulses are obtained by gluing together fronts and smallamplitude fronts.By Lemma 3.2, the extended point spectrum of the small-amplitude fronts in $\{\operatorname{Re} \lambda \geq 0\}$ is empty. Using this fact, the conclusion of the lemma is now a consequence of the gluing result in [27], which asserts that the extended point spectrum at glued heteroclinics converges to the union of extended point spectra at individual heteroclinics.

Lemma 3.4 The extended point spectrum in $\Sigma$ consists of precisely one real eigenvalue $\lambda_{*}$, which is algebraically simple. The eigenvalue is located at the origin on the curves of excitation pulses and steep fronts, only.

Proof. Continuity of the extended point spectrum, Lemma 3.3, guarantees that we find precisely the continuation of the zero eigenvalue of the steep pulse. The eigenvalue is located at the origin if and only if we have a front or pulse that decays exponentially as $\xi \rightarrow \infty$ with rate at least $\eta>0$, small but fixed. This is the case precisely for steep fronts and excitation pulses, not for the slow and fast fronts, which decay exponentially with rate $\nu_{0}=\mathrm{O}(\sqrt{\mu})$.

Corollary 3.5 Fronts with speeds between the speed of the excitation pulse and the speed of the steep front are either all unstable or stable. Similarly, all other fronts and algebraic pulses are all either stable or unstable. 
We now show that the zero eigenvalue actually crosses the origin with non-vanishing speed as $c$ increases through the speed of the steep front. Moreover, we compute the sign of the crossing speed and show that it is given by an interaction coefficient.

Lemma 3.6 The eigenvalue $\lambda_{*}$ in the extended point spectrum close to the origin depends smoothly on $c$ for fronts.

Proof. We need to show that the front profile is smooth in $c$. This can be readily seen by continuing the fronts with the implicit function theorem and a phase condition, using that the linearization at fronts is onto, Fredholm with index 1, in spaces of bounded, uniformly continuous functions. See, for instance, [14] for more details.

We denote by $e(\xi)$ and $e^{*}(\xi)$ the kernel of $\mathcal{L}$ in $L_{\eta}^{2}$ and its $L^{2}$-adjoint $\mathcal{L}^{*}$ in $L_{-\eta}^{2}$, respectively, and assume the normalization $\left(e, e^{*}\right)_{L^{2}}=1$, throughout. We can continue $e$ in $c$ in a smooth fashion as follows. We set $e(\xi ; c):=\partial_{\xi} u_{\mu, c}(\xi)$, the derivative of the front solutions. Note however that $e(\xi ; c)$ does not belong to the kernel of $\mathcal{L}$ in $L_{\eta}^{2}$ for $c \neq c_{\mathrm{sf}}$. We have the following lemma on the asymptotics.

Lemma 3.7 Let $\delta>0$ be as in Hypothesis 1.3. Also, let $\nu_{0}=\nu_{0}(\mu, c)$ be the eigenvalue of the linearization of the traveling-wave equation inside of the center-manifold at $u_{+}$, hence $0>\nu_{0}=\mathrm{O}(\sqrt{|\mu|})$. Then we have the following asymptotic expansions:

$$
\begin{aligned}
\partial_{c} e(\xi) & =e_{c, \infty} \mathrm{e}^{\nu_{0} \xi}+\mathrm{O}\left(\mathrm{e}^{-\delta \xi}\right), \\
\partial_{\xi c} e(\xi) & =\nu_{0} e_{c, \infty} \mathrm{e}^{\nu_{0} \xi}+\mathrm{O}\left(\mathrm{e}^{-\delta \xi}\right), \\
e^{*}(\xi) & =e_{\infty}^{*} \mathrm{e}^{-\nu_{0} \xi}+\mathrm{O}\left(\mathrm{e}^{-\delta \xi}\right), \\
\partial_{\xi} e^{*}(\xi) & =-\nu_{0} e_{\infty}^{*} \mathrm{e}^{-\nu_{0} \xi}+\mathrm{O}\left(\mathrm{e}^{-\delta \xi}\right),
\end{aligned}
$$

for $\xi \rightarrow \infty$. The nonzero vectors $e_{c, \infty}$ and $e_{\infty}^{*}$ belong to the kernels of $D \nu_{0}^{2}+c \nu_{0}+\partial_{u} f$ and $D \nu_{0}^{2}-c \nu_{0}+\left(\partial_{u} f\right)^{T}$, respectively, where $c, \mu$, and $\partial_{u} f$ are evaluated at a steep front at $\xi=+\infty$. For $\xi \rightarrow-\infty, \partial_{c} e$ and $\partial_{c \xi}$ e are bounded, while $e^{*}$ and $\partial_{\xi} e^{*}$ decay exponentially with uniform rate $\mathrm{O}\left(\mathrm{e}^{\delta \xi}\right)$.

Proof. The estimates follow in a straightforward fashion from estimates on asymptotics of solutions to linear, nonautonomous differential equations, as for instance presented in [8]. The function $e$ is the first component of a solution to the linearized traveling-wave ODE and derivatives with respect to $c$ satisfy inhomogeneous linear ODEs. We therefore have exponential expansions of the form described in (3.1). We need to establish non-vanishing of the leading-order coefficients. First, not that transverse crossing in [7] establishes that $e_{c, \infty}$ does not vanish. We need a short piece of algebra to understand the limit of $e^{*}$ : one readily verifies that $\Psi=\left(c e^{*}-D \partial_{\xi} e^{*}, D e^{*}\right)$ satisfies the adjoint ODE-linearization

$$
\psi_{1}^{\prime}=\left(\partial_{u} f\right)^{T} D^{-1} \psi_{2}, \quad \psi_{2}^{\prime}=-\psi_{1}+D^{-1} c \psi_{2} .
$$

Since $e^{*}, \partial_{\xi} e^{*} \in L_{-\eta}^{2}$, but $e^{*} \notin L_{\eta}^{2}$ (by invertibility of $\mathcal{L}$ in $L_{-\eta}^{2}$, Hypothesis 1.3), $\Psi$ cannot be bounded. It grows with the eigenvalue $-\nu_{0} \sim 0$ of the adjoint linearization $D \nu_{0}^{2}-c \nu_{0}+\left(\partial_{u} f\right)^{T}$ at $u_{+}$. Again, the precise asymptotics are a consequence of [8]. 
Lemma 3.8 The derivative of the eigenvalue $\lambda_{*}=0$ at the steep front with respect to $c$ is given through

$$
\partial_{c} \lambda_{*}=c_{*} e_{\infty}^{*} \cdot e_{c, \infty}+\mathrm{O}(\sqrt{\mu}),
$$

where we assumed $\left(e^{*}, e\right)_{L^{2}}=1$, and $\nu_{0}, e_{\infty}^{*}$, and $e_{c, \infty}$ were introduced in Lemma 3.7.

Proof. By regular perturbation theory, we have

$$
\partial_{c} \lambda_{*}=\frac{\left(e^{*},\left(\partial_{c} \mathcal{L}\right) e\right)_{L^{2}}}{\left(e^{*}, e\right)_{L^{2}}},
$$

where $e, e^{*}$ are kernel and adjoint kernel of the linearization at the steep front. Some attention needs to be paid to the fact that we are working in $L_{\eta}^{2}$ and adjoints must be computed appropriately. We will always use the $L^{2}$-inner product, so that adjoints are defined on $L_{-\eta}^{2}$. In particular, $e$ is given by the derivative of the steep front. Considering $\mathcal{L}$ as a differential operator, not taking into account function spaces for the time being, and writing $e=e(c)$ for the derivative of the fronts, we have $\mathcal{L} e=0$ for all $c$, so that $\partial_{c}(\mathcal{L} e)=0$, and therefore, pointwise in $\xi,\left(\partial_{c} \mathcal{L}\right) e=-\mathcal{L}\left(\partial_{c} e\right)$. This implies

$$
\partial_{c} \lambda_{*}=-\frac{\left(e^{*}, \mathcal{L}\left(\partial_{c} e\right)\right)_{L^{2}}}{\left(e^{*}, e\right)_{L^{2}}}
$$

Since $e^{*}$ annihilates the range of $\mathcal{L}$, one is tempted to conclude that the right-hand side vanishes. Note however that $\partial_{c} e$ does not belong to $L_{\eta}^{2}$ ! The strategy in the following is nevertheless to integrate by parts in the numerator and find $\left(\mathcal{L}^{*} e^{*}, \partial_{c} e\right)_{L^{2}}$, which vanishes. We will however generate nontrivial boundary terms. More precisely, we find

$$
\left(e^{*}, \mathcal{L}\left(\partial_{c} e\right)\right)_{L^{2}}=\left(\mathcal{L}^{*} e^{*}, \partial_{c} e\right)_{L^{2}}+\left[e^{*}, D \partial_{\xi} \partial_{c} e\right]_{-\infty}^{+\infty}-\left[\partial_{\xi} e^{*}, D \partial_{c} e\right]_{-\infty}^{+\infty}+c\left[e^{*}, \partial_{c} e\right]_{-\infty}^{+\infty} .
$$

In evaluating the brackets at the limits $\xi= \pm \infty$, we already used that these products actually converge by Lemma 3.7. Substituting the expressions in (3.1), and using that $\mathcal{L}^{*} e^{*}$ vanishes identically, we find

$$
\left(e^{*}, \mathcal{L}\left(\partial_{c} e\right)\right)_{L^{2}}=-2 \nu_{0} e_{\infty}^{*} \cdot D e_{c, \infty}+c e_{\infty}^{*} \cdot e_{c, \infty} .
$$

Since both $\nu_{0}=\mathrm{O}(\sqrt{\mu})$ and $c-c_{*}=\mathrm{O}(\sqrt{\mu})$, this proves the lemma.

We will show in Section 3.4 that the scalar product of these vectors converges to a nonzero limit as $\mu \rightarrow 0$ and is close to a quantity that defines the sign of interaction forces between excitation pulses. We can then conclude that actually $\partial_{c} \lambda_{*}<0$.

\subsection{Trigger waves}

We use [30] to determine stability of the trigger waves. Similarly to Lemma 3.7, we have the following asymptotics. 
Lemma 3.9 For the linearization at the excitation pulse, denote the kernel vector, given by the derivative of the excitation pulse, by $e_{\mathrm{p}}$, the adjoint kernel vector by $e_{\mathrm{p}}^{*}$, and normalize $\left(e_{\mathrm{p}}^{*}, e_{\mathrm{p}}\right)_{L^{2}}=1$. We then have the following expansions:

$$
\begin{aligned}
e_{\mathrm{p}}(-\xi) & =e_{\mathrm{p},-\infty} \mathrm{e}^{-\nu_{0} \xi}+\mathrm{O}\left(\mathrm{e}^{-\delta \xi}\right), \\
e_{\mathrm{p}}^{*}(\xi) & =e_{\mathrm{p}, \infty}^{*} \mathrm{e}^{-\nu_{0} \xi}+\mathrm{O}\left(\mathrm{e}^{-\delta \xi}\right),
\end{aligned}
$$

for $\xi \rightarrow \infty$. Here, $\delta>0$ is the $\mu$-independent spectral gap bound defined in Hypothesis 1.3 The decay rate $\nu_{0}>0$ is the small unstable eigenvalue of $u_{-}$inside the center-manifold of the traveling-wave $O D E$. The vectors $e_{\mathrm{p},-\infty}$ and $e_{\mathrm{p}, \infty}^{*}$ belong to the kernel of $D \nu_{0}^{2}+c \nu_{0}+\partial_{u} f$ and $D \nu_{0}^{2}-c \nu_{0}+\left(\partial_{u} f\right)^{T}$, respectively, where $c, \mu$, and $\partial_{u} f$ are evaluated at background state of the excitation pulse.

Theorem 4 [30] Define

$$
F_{\text {int }}^{\infty}:=e_{\mathrm{p}, \infty}^{*} \cdot D e_{\mathrm{p},-\infty} .
$$

Then the trigger waves are spectrally stable if $F_{\mathrm{int}}^{\infty}<0$ and spectrally unstable if $F_{\mathrm{int}}^{\infty}>0$. Moreover, we have normal dispersion $c^{\prime}(L)<0$ for spectrally stable wave trains and anomalous dispersion $c^{\prime}(L)>0$ for spectrally unstable wave trains. Here $c(L)$ denotes the wave speed of trigger waves with wavelength $L$.

Proof. The stability statement is a consequence of [30, Cor. 5.1]. The character of the dispersion relation follows from [30, Theorem 4.1]. In fact, (78) in [30] with the assumptions of $[30$, Cor. 5.1] reduces to

$$
\left(v_{0}, w_{0}\right) \mathrm{e}^{-2 \nu_{0} L}-M c=\mathrm{O}\left(\mathrm{e}^{-\delta L}\right),
$$

where we set $\mu=c$, and $v_{0}=\left(e_{\infty},-\nu_{0} e_{\infty}\right)^{T}$ and $w_{0}=\left(e_{\infty}^{*}, \nu_{0} e_{\infty}^{*}\right)^{T}$ are the leading order limiting vectors of the unique bounded solutions to the linearized equation at $-\infty$ and $+\infty$, respectively. Comparing this expression with the expansion (89) for $\lambda(\gamma)$, one readily concludes that attractive interaction corresponds to $c^{\prime}(L)>0$ and repulsive interaction to $c^{\prime}(L)<0$.

Remark 3.10 We remark that

$$
\operatorname{sign}\left(e_{\mathrm{p}, \infty}^{*} \cdot D e_{\mathrm{p},-\infty}\right)=\operatorname{sign}\left(e_{\mathrm{p}, \infty}^{*} \cdot e_{\mathrm{p},-\infty}\right),
$$

because for $\mu=0, e_{\mathrm{p}, \infty}^{*}$ and $e_{\mathrm{p},-\infty}$ belong to the kernel of $\partial_{u} f$ and $\partial_{u} f^{T}$, evaluated at the background state of the steep pulse, respectively, and we assumed that $e_{0}^{*} \cdot D e_{0}>0$ for any $e_{0}$ in the kernel and $e_{0}^{*}$ in the cokernel with $e_{0}^{*} \cdot e_{0}=1$; see Hypothesis 1.1, (iii). Continuity gives the sign relation for $\mu<0$, as claimed.

\subsection{The link between excitation pulses and steep fronts}

In this section we prove Theorem 2 up to the statements on algebraic pulses and phase waves. 
Lemma 3.11 The crossing speed of the zero eigenvalue at the steep front, computed in Lemma 3.8, is always negative, $\partial_{c} \lambda_{*}<0$.

Proof. Recall from Lemma 3.8 that

$$
\partial_{c} \lambda_{*}=c_{*} e_{\infty}^{*} \cdot e_{c, \infty}+\mathrm{O}(\sqrt{|\mu|}) .
$$

In fact, $e^{*}$ and also $e_{\infty}^{*}$ vary continuously in $\mu$ and converge as $\mu \rightarrow 0$. The same holds for $e_{\mathrm{p}}^{*}$ and $e_{\mathrm{p},-\infty}^{*}$ associated with the pulse. Moreover, $e^{*}(\xi) /\left|e^{*}(\xi)\right|$ approaches a nonzero limit $e_{\mathrm{sp}}^{*}$ as $\xi \rightarrow \infty$ up to $\mu=0$, and this limit coincides for pulses and steep fronts; it is given by the corresponding limit for the steep pulse. Again, this follows from the expansions for the associated ordinary differential equation in [8]. Similarly, $e_{c, \infty}$ approaches a limit at $\mu \rightarrow 0$, and at $\mu=0$ we have $e_{\infty}^{*} \cdot e_{c, \infty} \neq 0$, and $e_{\mathrm{p},-\infty}$ approaches a limit $e_{-\infty}$ as $\mu \rightarrow 0$.

We orient the kernel of $\partial_{u} f$,evaluated at $u=0, \mu=0$, such that $e_{0}:=\lim _{\xi \rightarrow-\infty} e(\xi) /|e(\xi)|$, where $e$ is the derivative of the steep pulse. In order to prove the lemma, we now assume that $\partial_{c} \lambda_{*}>0$ at $\mu=0$, that is, $e_{\infty}^{*} \cdot e_{c, \infty}>0$ since $c_{*}>0$, and find a contradiction. To fix orientations, let us also assume for now that $e_{\infty}^{*} \cdot e_{0}>0$, the other case being analogous; see Figure 3.1. Inspecting the bifurcation diagram, we conclude that pulses then exist for speeds



Figure 3.1: The diagram shows the vectors $e_{\infty}^{*}, e_{c, \infty}, e_{0}$, and the direction of motion of the intersection between unstable manifold and strong stable foliation upon variation of $c$, assuming that $\partial_{c} \lambda>0$.

less than the speed of the steep front, and trigger waves are stable with normal dispersion, which implies $F_{\text {int }}^{\infty}<0$. Indeed, Figure 3.1 shows the motion of the intersection point between unstable manifold and strong stable foliations relative to the vectors $e_{0}, e_{\infty}^{*}$ and $e_{c, \infty}$. Note that these vectors are vectors in $\mathbb{R}^{N}$ while the ODE dynamics relate to the phase space $\mathbb{R}^{2 N}$, but ODE eigenvectors and adjoint eigenvectors to the small eigenvalues on the center manifold are uniquely determined by their first component, so that the vectors plotted here point in directions such that their first component coincides with the vectors $e_{0}, e_{\infty}^{*}$ and $e_{c, \infty}$, respectively. Also note that the strong stable leave selected by the center-unstable manifold moves to the left when $c$ increases since $e_{c, \infty} \cdot e_{\infty}^{*}>0$. Now, by Remark 3.10, and by the convention $e_{\mathrm{p},-\infty} \rightarrow e_{-\infty}=e_{0}$

$$
\operatorname{sign} F_{\mathrm{int}}^{\infty}=\operatorname{sign}\left(e_{\infty}^{*} \cdot e_{-\infty}\right)=\operatorname{sign}\left(e_{\infty}^{*} \cdot e_{0}\right)>0 .
$$

which contradicts our initial assumption. 
If on the other hand $e_{\infty}^{*} \cdot e_{0}<0$, we find pulses faster than the steep front and anomalous dispersion with unstable trigger waves, so that $F_{\text {int }}^{\infty}>0$. This time, Remark 3.10 gives

$$
\operatorname{sign} F_{\text {int }}^{\infty}=\operatorname{sign}\left(e_{\infty}^{*} \cdot e_{-\infty}\right)=\operatorname{sign}\left(e_{\infty}^{*} \cdot e_{0}\right)<0,
$$

which again contradicts our assumption. As a consequence, $\partial_{c} \lambda_{*}>0$ is impossible, and we have proved the lemma.

Remark 3.12 With the normalizations chosen, we saw that attractive interaction of excitation pulses, $\iota=1$, corresponds to $e_{\infty}^{*} \cdot e_{0}>0$, so that in fact

$$
\iota=\operatorname{sign}\left(e_{\infty}^{*} \cdot e_{0}\right) .
$$

\subsection{Algebraic pulses and phase waves}

We conclude the proof of Theorem 2. The algebraic pulses are limits of fronts in $L^{\infty}$ in both bifurcation diagrams. Continuity of the point spectrum, Lemma 3.1, implies that the point spectrum of the algebraic pulse is the limit of point spectra of the fronts in $\operatorname{Re} \lambda \geq-\delta_{0}$ for some $\delta_{0}>0$. Here, $\delta_{0}$ refers to a bound on the real part of the essential spectrum in fixed exponentially weighted space $L_{\eta}^{2}, \eta>0$. The point spectrum therefore consists of precisely one eigenvalue, $\lambda_{*} \leq 0$ in the case of repulsive interaction, and $\lambda_{*} \geq 0$ in the case of attractive interaction. On the other hand, $\lambda=0$ cannot be an eigenvalue in $L_{\eta}^{2}$, since the algebraic pulse is not exponentially decaying at $\xi=+\infty$. This shows that the algebraic pulse is unstable for attractive interaction and stable for repulsive interaction.

We turn to the phase waves near the algebraic pulse, next.

Lemma 3.13 There is $L_{0}>0$ such that for all $L>L_{0}$, the spectrum of a phase wave in $L_{\eta}^{2}$ near a fixed algebraic pulse with wavelength $L$ consists precisely of a circle of essential spectrum in $\operatorname{Re} \lambda \geq-\delta_{0}$. The circle converges to the eigenvalue of the algebraic pulse as $L \rightarrow \infty$.

Proof. The proof is a consequence of [15]. The linearization at the algebraic pulse in the exponentially weighted space $L_{\eta}^{2}$ consists of point spectrum only for $\operatorname{Re} \lambda>-\delta_{0}$. Wave trains of period $L$ are close to an algebraic pulse on any finite interval of length $L$. Gardner [15] shows that the spectrum of a wave train on $\xi \in \mathbb{R}$ is the union of the spectra on $[0, L]$, equipped with Floquet boundary conditions for the eigenfunctions, $u(L)=\mathrm{e}^{\mathrm{i} \gamma} u(0), u_{\xi}(L)=\mathrm{e}^{\mathrm{i} \gamma} u_{\xi}(0)$. The union is taken over all Floquet (or Bloch wave) exponents $\gamma \in[0,2 \pi)$. He then shows that each of the Floquet eigenvalue problems possesses spectrum close to the point spectrum of the pulse as $L \rightarrow \infty$. In other words, one will find a circle of spectrum parametrized by $\gamma \in[0,2 \pi)$ near each eigenvalue of the algebraic pulse. Also, the linearization near the wave train will be invertible for values of $\lambda$ where the linearization along the pulse is invertible; see again [15]. While the analysis in [15] is carried out in $L^{2}$, introducing a small exponential weight does not change statements or arguments. This proves the lemma.

This concludes the proof of Theorem 2 . 


\section{Examples}

\subsection{A scalar phase model}

The simplest model problem is the scalar phase equation

$$
\theta_{t}=\theta_{x x}+f(\theta), \quad f(\theta)=f(\theta+2 \pi) .
$$

Traveling-wave profiles $\theta(x-c t)$ can be found using phase-plane analysis for

$$
\theta_{\xi}=v, \quad v_{\xi}=-c v-f(\theta) .
$$

A convenient specific choice is $f(\theta ; \mu)=\cos (\theta)-1-\mu$. Ermentrout and Rinzel [12] studied this system and essentially recovered our bifurcation diagram with repulsive interaction for $\iota<0$; see Figure 4.1. In fact, when $f(0)=f^{\prime}(0)=0, f^{\prime \prime}(0)>0$, one can use a shooting method to establish existence of a heteroclinic (homoclinic once $\theta=0$ and $\theta=2 \pi$ are identified) orbit connecting $\theta=0$ and $\theta=2 \pi$, which converges exponentially as $\xi \rightarrow+\infty$. In fact, one can even obtain stability information fairly easily in this situation: all monotone profiles (now with $\theta$ considered as a function in $\mathbb{R}$ ) are stable and all non-monotone profiles are unstable. Phase- and trigger waves are all stable, slow fronts are non-monotone and unstable. This can be readily seen by inspecting the linearization, which is a Sturm-Liouville problem with eigenvalue zero and eigenfunction given by the derivative of the wave profile. Since the first eigenfunction (when it exists, that is, when the most unstable eigenvalue is not contained in the essential spectrum) of scalar eigenvalue problems has a sign. This shows that the monotone pulses and steep fronts, with derivative belonging to the point spectrum, are stable. A slightly more subtle consideration of the winding of the $\operatorname{angle} \arctan \left(u^{\prime}(\xi) / u(\xi)\right)$ for solutions to the eigenvalue ODE then shows that non-monotone slow fronts are unstable and monotone fast fronts are stable; see also [16]. Linearization at wave trains consists of essential spectrum, only. The Bloch-wave decomposition described in Lemma 3.13 does however allow us to view the spectrum as the closure of the spectrum on finite periodic domains with period $k L, k \in \mathbb{N}$. On such periodic domains, the above Sturm-Liouville argument applies once again, and we can conclude that monotone waves are (spectrally) stable while non-monotone waves are unstable.

One can actually obtain somewhat more information in this scalar case, going beyond the local unfolding near $c=c_{*}$. In particular, one finds that the phase waves can be continued for any fixed $\mu \geq 0$ in the period $L$, with monotonically increasing speed $c$. In the limit $L \rightarrow \infty$, the speed grows linearly in $k$ with slope $c \sim \omega_{*} L$. The limiting wave is the spatially homogeneous oscillation with frequency $\omega_{*} \sim \sqrt{\mu}$; see $[12,6]$.

\subsection{FitzHugh-Nagumo and variations}

Examples of traveling pulses for which existence and stability can be shown analytically are rare. The Fitz-Hugh Nagumo equation (1.2) with $\varepsilon$ small might well be the most prominent one. The transition from excitable to oscillatory behavior can be observed as $\rho$ is varied, which however does not entrain any steady-state bifurcations in the kinetics. Instead of a saddlenode, the kinetics exhibit a Hopf bifurcation and, for small $\varepsilon$, quite complicated behavior of 




phase wave
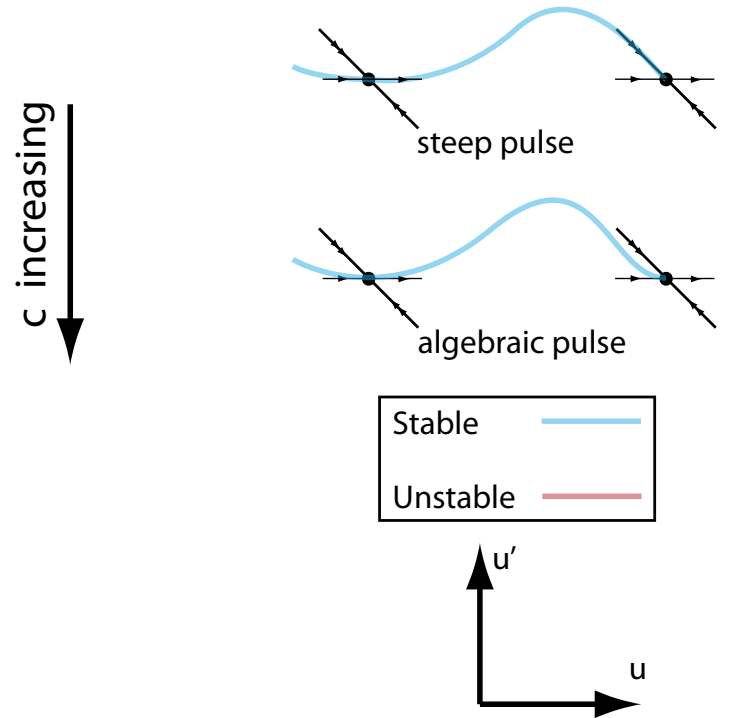
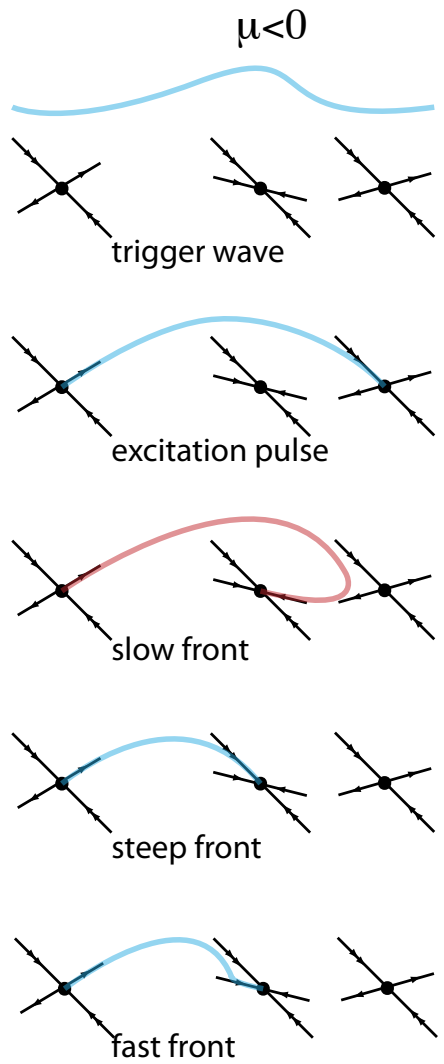

Figure 4.1: The heteroclinic bifurcation in (4.2) in the $\left(\theta, \theta_{\xi}\right)$-phase plane. 

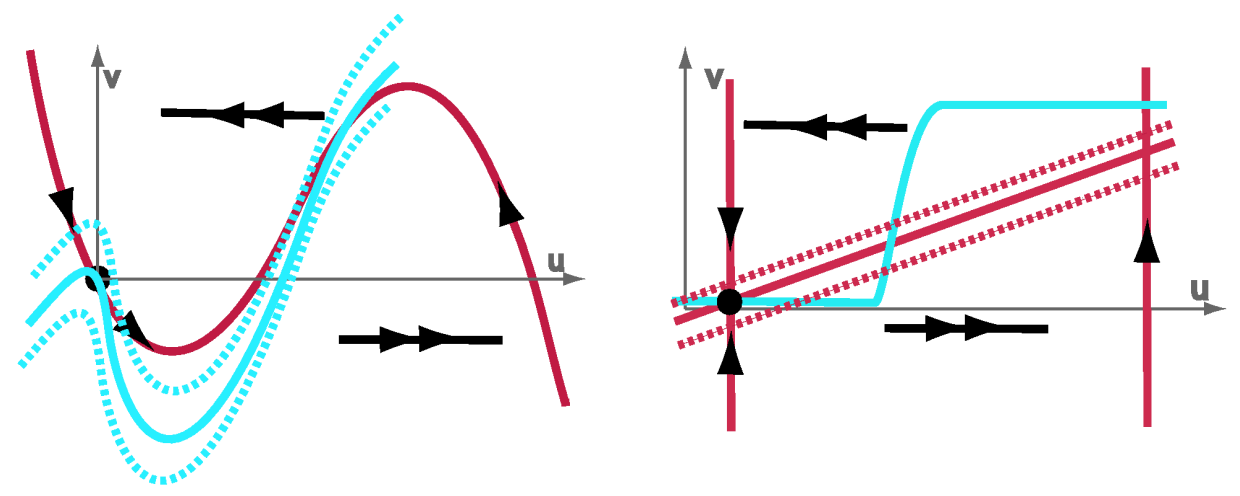

Figure 4.2: A sketch of nullclines at criticality for variants of the FitzHugh-Nagumo equation, which lead to transitions from excitable to oscillator behavior as analyzed in our main results.

periodic orbits; see the discussion, below. When one changes the shape of the $v$-nullcline, one can however construct examples where the excitation pulse limits on a saddle-node bifurcation in the kinetics; see Figure 4.2. Our main results would then apply to the excitation pulse when small diffusion of the inhibitor is present. Alternatively, one could also adapt our results in a straight-forward fashion to the case of vanishing diffusivity in the inhibitor. The main difference would be a slightly different general form of the traveling-wave equation since $D$ would not be invertible, and a different counting of Morse indices in Lemma 2.1.

A variant of the FitzHugh-Nagumo equation was introduced by Barkley [4] to enable fast simulations, and later modified in [2] as a model for CO-oxidation on platinum surfaces.

$$
\begin{aligned}
& u_{t}=u_{x x}+u(1-u)\left(u-\frac{b+v}{a}\right) \\
& v_{t}=\varepsilon(g(u)-v)
\end{aligned}
$$

where $g$ was assumed linear in [4] and $g(u) \equiv 0$ for $u<1 / 3$ in [2]. Varying $b \sim 0$, one observes a transcritical steady-state bifurcation in the kinetics, marking the transition from excitable $(b>0)$ to oscillatory $(b<0)$ behavior. One numerically observes that excitation pulses limit on a steep pulse for $\varepsilon>0$ fixed, small, as $b$ is decreased to zero. Although not stated here, this transverse bifurcation can be analyzed in a similar fashion, leading again to two bifurcation diagrams corresponding to attractive and repulsive pulse interaction; see also the discussion, below. On the other hand, small perturbations such as adding a small constant in the activator $u$-kinetics, will unfold the transcritical bifurcation into two saddle-node bifurcations, which correspond precisely to the scenario considered here.

The interaction behavior of pulses is actually somewhat subtle in this model. For fixed $b>0$, excitation pulses repel for $\varepsilon>0$, small enough. Bär and Or-Guil [21] observed that the interaction changes to attractive interaction when $\varepsilon$ is increased above some threshold value $\varepsilon_{\mathrm{c}}(b)$. We numerically found that $\varepsilon_{\mathrm{c}}(b) \rightarrow 0$ as $b \rightarrow 0$. As a consequence, we expect the transition scenario with attractive pulse interaction to occur when $b$ is varied and $\varepsilon>0$ is kept small but fixed. 


\section{Summary and outlook}

We presented a bifurcation and stability analysis for coherent structures at the boundary between excitable and oscillatory media. We argue that the simplest transition between the two types of media is organized around a steep pulse, which in a traveling-wave ODE corresponds to an orbit-flip homoclinic to a saddle-node equilibrium. Unfolding this codimension-two homoclinic orbit, we found various fronts, excitation pulses, trigger waves, phase waves, and a family of algebraic pulses. We were able to determine stability for most of the bifurcating waves in suitable function spaces. The main open question is to determine the stability of both trigger and phase waves in a full neighborhood of the bifurcation point. The stability information we gathered and the information from simple scalar models however suggest that no changes of stability occur in the region that is not covered by our result.

From a pathfollowing perspective, we uncovered two noteworthy points:

- trigger waves continue into phase waves without any notable change in stability;

- excitation pulses can be continued as steep fronts after a saddle-node at a steep pulse, but the saddle-node bifurcation does not entrain an exchange of stability in point spectrum.

Indeed, the steep fronts are stable in appropriate exponentially weighted spaces. The exchange of stability is in terms of essential spectrum in unweighted, $L^{2}$-spaces, only.

A notable difference between trigger and phase waves of course is the limiting behavior for large wavelengths. Trigger waves limit on excitation pulses with finite limiting speed, whereas the speed of phase waves diverges as the wavelength approaches infinity. Near the bifurcation point that we examined, all dispersion relations are monotone, governed by the interaction near the marginally stable equilibrium.

Another noteworthy, and somewhat surprising point, is that fast fronts are always stable. Indeed, this has been proven in a number of model problems and conjectured to hold true for a large class of spatially extended invasion problems [34]. The steep fronts are termed pushed fronts, there, since their speed is determined by the nonlinear profile of the front, rather than by the linear invasion speed, which depends on the linearization at the unstable equilibrium, only. We found it somewhat surprising to find this somewhat global (in $c$ ) stability information in our local $\left(c \sim c_{*}\right)$ stability analysis. In fact, it is quite impossible to determine the sign of $\partial_{c} \lambda_{*}$ in Lemma 3.8 directly, without the additional information on pulses and pulse trains that are nearby.

We also emphasize that in our case it is not true that the steep front is the slowest stable front: in fact, there is a one-parameter family of fronts, the small fronts, found as heteroclinic

orbits inside the center-manifold, which are stable for values $c \geq c_{\text {lin }}=\mathrm{O}(\sqrt{|\mu|})$, the linear invasion speed.

Our results naturally extend to pitchfork and transcritical bifurcations as considered in [9]. Inspecting the bifurcation diagrams, there, one can readily fill in the stability information with the guidance of our main stability result, Theorem 2. A more challenging open problem is the analysis of Hopf bifurcations in the traveling-wave ODE. 
We suspect that our results could be extended beyond a stability analysis of coherent structures by proving an interaction theorem for pulses and small fronts for $\mu \sim 0$. A rigorous interaction theorem would however require a substantial extension of the analysis in [36].

\section{References}

[1] J. Alexander, R. Gardner, and C. Jones. A topological invariant arising in the stability analysis of travelling waves. J. Reine Angew. Math. 410 (1990), 167-212.

[2] M. Bär, N. Gottschalk, M. Eiswirth, and G. Ertl. Spiral waves in a surface reaction: Model calculations. J. Chem. Phys. 100 (1994), 1202-1215.

[3] M. Bär and M. Or-Guil. Alternative scenarios of spiral breakup in a reaction-diffusion model with excitable and oscillatory dynamics. Phys. Rev. Lett. 82 (1999), 1160-1163.

[4] D. Barkley. A model for fast computer simulation of waves in excitable media. Physica D 49 (1991), 61-70.

[5] G. Bordiougov and H.Engel. From trigger to phase waves and back again. Phys. D 215 (2006), 25-37.

[6] J. Bellay. The stability and transitions of coherent structures on excitable and oscillatory media. PhD Thesis, University of Minnesota, 2008.

[7] S.-N. Chow and X.-B. Lin. Bifurcation of a homoclinic orbit with a saddle-node equilibrium. Differential Integral Equations 3 (1990), 435-466.

[8] E. Coddington and N. Levinson. Theory of ordinary differential equations. McGraw-Hill Book Company, Inc., New York-Toronto-London, 1955.

[9] B. Deng. Homoclinic bifurcations with nonhyperbolic equilibria. SIAM J. Math. Anal. 21 (1990), 693-720.

[10] A. Doelman, B. Sandstede, A. Scheel, and G. Schneider. The dynamics of modulated wave trains. Memoirs of the AMS, 199 (2009).

[11] S.-I. Ei. The motion of weakly interacting pulses in reaction-diffusion systems. J. Dynam. Differential Equations 14 (2002), 85-137.

[12] G. Ermentrout and J. Rinzel. Waves in a simple, excitable or oscillatory, reactiondiffusion model. J. Math. Biol. 11 (1981), 269-294.

[13] B. Fiedler and A. Scheel. Spatio-Temporal Dynamics of Reaction-Diffusion Patterns. In Trends in Nonlinear Analysis, M. Kirkilionis, S. Krmker, R. Rannacher, F. Tomi (Eds.), Springer-Verlag, Berlin, 2003.

[14] M. Friedman and E. Doedel. Numerical computation and continuation of invariant manifolds connecting fixed points. SIAM J. Numer. Anal. 28 (1991), 789-808. 
[15] R. Gardner. Spectral analysis of long wavelength periodic waves and applications. J. Reine Angew. Math. 491 (1997), 149-181.

[16] P. Hagan, The instability of nonmonotonic wave solutions of parabolic equations, Stud. Appl. Math. 64 (1981), 57-88.

[17] D. Henry. Geometric theory of semilinear parabolic equations. Lecture Notes in Mathematics, 840. Springer-Verlag, Berlin-New York, 1981.

[18] A.J. Homburg. Global aspects of homoclinic bifurcations of vector fields. Memoirs of the A.M.S. 578, (1996).

[19] R. Kollár, A. Scheel Coherent structures generated by inhomogeneities in oscillatory media. SIAM J. Appl. Dyn. Syst. 6 (2007), 236-262.

[20] J. Moehlis. Canards in a surface oxidation reaction. J. Nonlinear Sci. 12 (2002), 319-345.

[21] M. Or-Guil, I.G. Kevrekidis, and M. Bär. Stable bound states of pulses in an excitable medium. Phys. D 135 (2000), 154-174.

[22] K. Promislow. A renormalization method for modulational stability of quasi-steady patterns in dispersive systems. SIAM J. Math. Anal. 33 (2002), 1455-1482.

[23] J. Rademacher, A. Scheel Instabilities of wave trains and Turing patterns in large domains Int. J. Bif. Chaos 16 (2007), 2679-2691.

[24] J. Rademacher and A. Scheel. The saddle-node of nearly homogeneous wave trains in reaction-diffusion systems. J. Dyn. Diff. Eqns. 19 (2007), 479-496.

[25] B. Sandstede. Center manifolds for homoclinic solutions. J. Dyn. Diff. Eqns. 12 (2000), 449-510.

[26] B. Sandstede. Stability of travelling waves. In: Handbook of Dynamical Systems II (B Fiedler, ed.). North-Holland (2002), 983-1055.

[27] B. Sandstede and A. Scheel. Gluing unstable fronts and backs together can produce stable pulses. Nonlinearity 13 (2000), 1465-1482.

[28] B. Sandstede and A. Scheel. Absolute versus convective instability of spiral waves. Phys. Rev. E. 62 (2000), 7708-7714.

[29] B. Sandstede, A. Scheel Absolute and convective instabilities of waves on unbounded and large bounded domains. Physica D 145 (2000), 233-277.

[30] B. Sandstede and A. Scheel. On the stability of periodic travelling waves with large spatial period. J. Diff. Eqns. 172 (2001), 134-188.

[31] B. Sandstede and A. Scheel. Defects in oscillatory media: toward a classification. SIAM J. Appl. Dyn. Syst. 3 (2004), 1-68. 
[32] B. Sandstede and A. Scheel. Relative Morse indices, Fredholm indices, and group velocities. Discr. Cont. Dyn. Sys. 20 (2008), 139-158.

[33] D. Sattinger. Weighted Norms for the Stability of Travelling Waves. J. of Diff. Eqn. 25 (1977), 130-144.

[34] W. van Saarloos. Front propagation into unstable states. Phys. Rep. 386 (2003), 29-222.

[35] M. Shashkov and D. Turaev. An existence theorem of smooth nonlocal center manifolds for systems close to a system with a homoclinic loop. J. Nonlinear Sci. 9 (1999), 525-573.

[36] S. Zelik and A. Mielke. Multi-pulse evolution and space-time chaos in dissipative systems. Mem. Amer. Math. Soc. 198 (2009), no. 925. 